analyse identisch mit dem früher aus Phenylrhodanid und Chloral hergestellten Kondensationsprodukt, dem $\beta, \beta, \beta-$ Trichlor- $\alpha, \alpha$-bis-[S-phenyl-thiocarbamido]-äthan ${ }^{5}$.

$$
\begin{gathered}
\text { Versuch } 3 \\
\text { Versuchte Umsetzung von }
\end{gathered}
$$

Phenylthiocarbamat mit Chloral

$3,05 \mathrm{~g}(0,02 \mathrm{Mol})$ Phenylthiocarbamat wurden bei $0^{\circ}$ in $20 \mathrm{ml}$ konz. $\mathrm{H}_{2} \mathrm{SO}_{4}$ unter Rühren gelöst. Nach $2 \mathrm{Stdn}$. wurde die homogene Lösung mit $1,95 \mathrm{~g}(0,02 \mathrm{Mol})$ Chloral versetzt und noch 5 Stdn. bei $0^{\circ}$ gerührt. Die nun schwach gelb gefärbte Lösung gaben wir in fein zerstoßenes Eis, wobei sich 3,0 g unverändertes Phenylthiocarbamat abschieden.

\section{Versuch 4}

U V-spektroskopische Messungen

Die Messungen wurden in einem BeckmannSpektrophotometer durchgeführt, und zwar im allgemeinen bei Zimmertemperatur. Nur die Messungen des UVSpektrums von $p$-Chlor-rhodanbenzol (I) in konz. $\mathrm{H}_{2} \mathrm{SO}_{4}$ mußten zwischen $0^{\circ}$ und $10^{\circ}$ ausgeführt werden, da sonst Zersetzungen eintraten. Um die Nullzeit-Kurve zu erhalten, wurde in folgender Weise verfahren: Sofort nach dem Herstellen der Lösung wurde die Absorption bei bestimmten Wellenlängen zusammen mit der Zeit (gerechnet vom Augenblick der Herstellung der Lösung) bestimmt. Dieser Vorgang wurde innerhalb einiger Stdn. mehrfach wiederholt, bis die Zeit als Funktion der Absorption graphisch dargestellt werden konnte. Die Kurve wurde dann auf die Nullzeit extrapoliert, so daß wir für jede untersuchte Wellenlänge die zur Nullzeit gehörende Absorption erhielten. Der ganze Vorgang wurde für andere Wellenlängen wiederholt, bis genügend Meßpunkte vorhanden waren.
Versuch 5

Beeinflussung der Thiocarbamatbildung durch 1.1-Diphenyl-äthanol

$2,0 \mathrm{~g}$ 1.1-Diphenyl-äthanol vom Schmp. $83^{\circ}$ und $10 \mathrm{ml}$ $\mathrm{CCl}_{4}$ wurden im Scheidetrichter mit $20 \mathrm{ml}$ konz. $\mathrm{H}_{2} \mathrm{SO}_{4}$ geschüttelt. Die tiefrot gefärbte Säurelösung wurde abgetrennt und in einer Eis-Kochsalz-Mischung auf $-5^{\circ}$ abgekühlt. Dann wurden tropfenweise $1,57 \mathrm{~g}(0,01 \mathrm{Mol})$ $n$-Heptylrhodanid zugesetzt. Nach 5-stdg. Rühren wurde die Lösung in ungefähr $200 \mathrm{~g}$ fein zerstoßenes Eis eingetragen. Die ausgeschiedenen Kristalle wurden abfiltriert, gewaschen und getrocknet: 3,4 g Rohprodukt. Bei einem entsprechenden Versuch ohne Zugabe von 1,57 $\mathrm{g} n$-Heptylrhodanid schieden sich beim Einrühren in Eiswasser 2,0 g unverändertes 1.1-Diphenyl-äthanol ab.

Ferner wurde der Versuch auch ohne Zusatz von 1.1Diphenyl-äthanol ausgeführt: Beim Einrühren in Eiswasser scheiden sich $0,2 \mathrm{~g} n$-Heptylthiocarbamat ab (wenn ebenfalls $1,57 \mathrm{~g} n$-Heptylrhodanid angewandt worden waren und die Versauchsdauer nur 5 Stdn. betrug).

Die beiden Kontrollversuche zeigen, daß sich das oben erhaltene Rohprodukt (3,4 g) aus 2,0 g 1.1-Diphenyl-äthanol

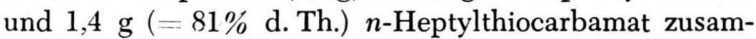
mensetzt. Beim Umkristallisieren des Rohproduktes aus Hexan schieden sich aus der heißen Lösung 1,35 g $n$ Heptylthiocarbamat vom Schmp. $103^{\circ}$ aus. 1.1-Diphenyläthanol blieb dagegen weitgehend in der Mutterlauge gelöst.

Der Deutschen Forschungsgemeinschaft sei für die Förderung der Arbeit bestens gedankt.

5 R. Riemschneider u. F. Wojahn, Pharmaz. 4, 406 [1949].

\title{
Eine Methode zur Ermittlung und Aufschreibung aller theoretisch möglichen Sessel-Konfigurationen für ein gegebenes Stellungsisomeres von Cyclohexan-Substitutionsprodukten ${ }^{1}$
}

\author{
Von Randolph Riemschneider ${ }^{2}$ \\ Aus der Freien Universität Berlin-Dahlem \\ (Z. Naturforschg. 10 b, 183-191 [1955]; eingegangen am 11. November 1954)
}

\begin{abstract}
Die zur Ermittlung und Aufschreibung aller zu einem gegebenen Stellungsisomeren gehörenden, theoretisch möglichen Sessel-Konfigurationen von Cyclohexan-Substitutionsprodukten entwickelte Methode wird erläutert und am Beispiel des 1.2.4.5-Tetrabrom-1.4-dichlorcyclohexans vorgeführt. Diese Methode ist nicht nur für Untersucher, die über CyclohexanSubstitutionsprodukte und verwandte Verbindungen arbeiten, von Interesse, sie hat insofern allgemeinere Bedeutung, als gezeigt wird, wie man vorgehen kann, wenn zu irgendeinem gegebenen Stellungsisomeren die theoretisch möglichen Konfigurationen gefunden werden sollen. Voraussetzung ist, da $\beta$ man über ein geeignetes Modell verfügt und die davon abgeleiteten Konfigurationen zeichnen und bezeichnen bzw. zeichnen oder bezeichnen kann.
\end{abstract}

$\mathrm{B}$ ei unseren Untersuchungen über den räumlichen Bau von Poly(hetero)halocyclohexanen und verwandten Verbindungen mußten wir häufig alle theoretisch möglichen Sessel-Konfigurationen für ein gegebenes Stellungs-

isomeres ermitteln und aufschreiben; die von uns verwendeten Konfigurations-Bezeichnungen und unsere besondere Art, Konfigurations-Tabellen aufzustellen, wurFußnoten 1 und 2 s. S. 184. 
den in Mitt. $\mathrm{XXX}^{3}$ und $\mathrm{XXI}^{4}$ dieser Reihe erläutert5. Das Ermitteln der Konfigurationen und das Aufschreiben von Sessel-Konfigurations-Bezeichnungen ist äußerst einfach, solange die zu einem Stellungsisomeren gehörende Anzahl der Konfigurationen klein ist. So läßt sich beispielsweise ohne weiteres übersehen, daß vom 1.4-Dibrom-cyclohexan folgende Sessel-Konfigurationen notiert werden können: 1e4e, 1e4a, 1a4a 5. Schwieriger wird es, wenn die Anzahl der theoretisch möglichen Konnfigurationen relativ groß ist, wie z. B. bei vielen CyclohexanSubstitutionsprodukten mit mehreren untereinander verschiedenen Substituenten, bei denen die Anzahl der Sessel-Konfigurationen bis auf 128 (einschließlich der Spiegelbilder) ansteigen kann. 128 ist die Höchstzahl der $\mathrm{zu}$ einem Stellungsisomeren gehörenden Sessel-Konfigurationen (Beispiele in Tab. 2, Ifd. Nr. 27, 29, 30, 35, 36 , 37). Bei Stellungsisomeren mit vielen zugehörigen Konfigurationen kommt es leicht vor, daß entweder nicht alle voneinander verschiedenen theoretisch möglichen Sessel-Konfigurationen notiert werden oder aber einige von diesen mehrmals, da sich für ein und dieselbe Konfiguration häufig mehrere Bezeichnungen aufschreiben lassen. Das Ermitteln der für ein bestimmtes Stellungsisomeres gesuchten Sessel-Konfigurationen und ihre Aufschreibung in Konfigurations-Tabellen erfordert also einige Übung. Die von uns bei der Anfertigung des Kataloges für Sessel-Konfigurationen von CyclohexanSubstitutionsprodukten mit einem bis zwölf untereinander gleichen Substituenten ${ }^{6}$ entwickelte Methode zur Aufstellung von Konfigurations-Tabellen soll im Folgenden erläutert und an einem Beispiel vorgeführt werden. Als Beispiel wurde ein hetero-substituiertes Cyclohexan gewählt, da für Cyclohexan-Substitutionsprodukte mit untereinander gleichen Substituenten ja ein Katalog ${ }^{6}$ zur Verfügung steht und damit die Möglichkeit gegeben ist, Konfigurations-Tabellen für alle Stellungsisomeren mit untereinander gleichen Substituenten einfach nachzuschlagen 7 .

Unsere Methode soll es Untersuchern, die über Cyclohexan-Substitutionsprodukte und verwandte Verbindungen arbeiten, erleichtern, für beliebige Stellungisomere die zugehörigen Konfigurationen abzuleiten und den unsrigen entsprechende Konfigurations-Tabellen ${ }^{8}$ aufzustellen.

\section{Allgemeines zur Methode}

Bei der zu besprechenden Methode gehen wir so vor, daß wir erstens durch Ermitteln und Aufschrei-

1 Fortsetzung der Mitt. XXIII der Reihe „Zur Chemie von Polyhalocyclohexanen und verwandten Verbindungen“: Z. Naturforschg. 8b, 701 [1953]; 9b, 244 [1954].

2 Anschrift für den Schriftverkehr: Berlin-Charlottenburg 9 , Bolivarallee 8 .

3 Osterr. Chemiker-Ztg. 55, 102, 161 [1954] 5

4 „Räumlicher Bau, Bezeichnung und Anzahl theoretisch möglicher Konfigurationen von Cyclohexan-Substitutionsprodukten“, Manuskript, auszugsweise veröffentlicht in Mitt. XXVIII ${ }^{14}, \mathrm{XXX}^{3}$ und l. c. ${ }^{6}$.

5 Verfasser verwendet jetzt die Buchstaben e und a an Stelle von e und p, seitdem auch die Autoren e und ben von Konfigurationen bzw. ihrer Bezeichnungen auf eine provisorische Tabelle hinarbeiten (a) und zweitens diese provisorische Tabelle in eine Form bringen, in der sie den Richtlinien für unsere SesselKonfigurations-Bezeichnungen entspricht (b), d. h. eine Konfigurations-Tabelle mit oder ohne getrennte Notierung optischer Antipoden aufstellen ${ }^{8}$.

Bei der Suche nach den Konfigurationen und geeigneten Bezeichnungen nehmen wir Zeichnungen zu Hilfe, da sich viele Überlegungen (besonders für den Ungeübten) an Hand von Zeichnungen leichter durchführen lassen als an Bezeichnungen. Wir gehen von „Bezeichnungen“ (Buchstabenkolonnen, Tafel 2) aus und übertragen jeweils die an Zeichnungen erkannten Zusammenhänge auf diese „Bezeichnungen“. In diesen „Bezeichnungen“ wird noch nicht angegeben, welche Substituenten sich oberhalb und welche sich unterhalb ihres zugehörigen C-Atoms befinden. In den verwendeten Zeichnungen, die in Abb. 1 erläutert sind, ist diese Angabe mit enthalten. Daher würden sich zu jeder „Bezeichnung“ jeweils zwei Zeichnungen anfertigen lassen [eine mit Kreis am Strich gefüllt am C-Atom $1(\bullet)$ und eine mit Kreis am Strich offen am C-Atom $1(\varphi)]$. Es genügt jedoch, jeweils nur eine Zeichnung anzufertigen, wenn am C-Atom 1 einheitlich [z. B. mit Kreis am Strich gefüllt $(\bullet)]$ begonnen wird. Aus diesen Zeichnungen sind die Symmetrieverhältnisse leicht ablesbar. Zum Beispiel geben Zeichnungen, die sich durch Drehen oder Klappen zur Deckung bringen lassen, wenn jeweils ein gefüllter Kreis auf einem offenen liegt und umgekehrt, optische Antipoden wieder.

a) Aufstellen einer provisorischen Konfigurations-Tabelle

Wir beginnen mit dem Aufschreiben aller Konfigurations-,,Bezeichnungen“, die sich für das als Beispiel gewählte Stellungsisomere durch Kombinie-

a schreiben, die e und $p$ eingeführt haben (C. W. B eckett, K. S. Pitzer, R. Spitzer, J. Amer. chem. Soc. 69, 2488 [1947]). - In vorliegender Mitteilung arbeiten wir ausschließlich mit dem Begriff „Konfiguration“, auch wenn man den Begriff „Konstellation“"verwenden könnte.

6 Tab. 3 der Mitt. XXI, 1. c. ${ }^{4}$. - Der Katalog wurde inzwischen in der japanischen Zeitschrift Sci. Insect Control (Botyu-Kagaku, Kyoto) 20, Heft 2 [1955] abgedruckt.

7 Für heterosubstituierte Cyclohexane wird man allerdings wegen der Fülle der Möglichkeiten keinesfalls einen vollständigen Katalog aufstellen.

8 Vgl. z. B. Tab. 2 bis 5 und 11 bis 16 der Mitt. XXX, l. c. ${ }^{3}$. 
ren der Buchstaben e und a finden lassen. Um beim Kombinieren unfehlbar alle aufschreibbaren „Bezeichnungen" "zu erhalten, hat sich eine ganz bestimmte Art des Aufschreibens als zweckmäßig erwiesen, die sich aus einer mehrmaligen Verwendung von Kombinationsquadraten ableiten läßt: Buchstabenkolonnen I, II, III, IV, V und VI der Tafel 2.

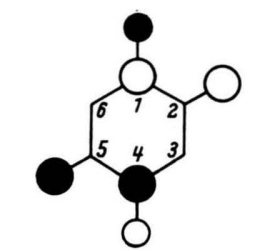

$1 \mathrm{a} 2 \mathrm{e} 4 \mathrm{a} 5 \mathrm{e}(\mathrm{Br}) 1 \mathrm{e} 4 \mathrm{e}(\mathrm{Cl})$

Abb. 1. Sessel-Konfiguration eines Cyclohexan-Substitutionsproduktes am Beispiel einer Konfiguration des 1.2.4.5-Tetrabrom-1.4-dichlor-cyclohexans.

Der Kohlenstoffring des Cyclohexan-Substitutionsproduktes ist als Sechseck dargestellt, die C-Atome sind im Uhrzeigersinn von 1 bis 6 numeriert. Die Substituenten werden durch Kreise symbolisiert, und zwar: $\mathrm{Br}$, großer Kreis: Cl, kleiner Kreis; H-Atome werden nicht mitgezeichnet. Die auf den Ecken des Sechsecks befindlichen Kreise bedeuten a-gebundene, die seitlich liegenden egebundene Substituenten (letztere durch Striche mit dem Sechseck verbunden). Substituenten, die sich oberhalb ihres zugehörigen C-Atoms befinden, werden durch gefüllte Kreise, solche, die sich unterhalb befinden, durch offene Kreise wiedergegeben. Vgl. auch Tafel 5 der Mitt. XXX dieser Reihe ${ }^{3}$.

Zeichnungen dieser Art dürfen nur in der Zeichenebene gedreht werden (d. h. um Achsen, die senkrecht zur Zeichenebene stehen). Beim Klappen (d.h. Drehen uin Achsen, die in der Zeichenebene liegen) kommen alle Substituenten, die oberhalb ihres zugehörigen C-Atoms gelegen haben, nach unten und umgekehrt, d.h. alle offenen Kreise sind nach dem Klappen gefüllt zu zeichnen und umgekehrt.

In welcher Art diese Buchstabenkolonnen zu verwenden sind, ist den Erläuterungen und den Beispielen zur Tafel $2 \mathrm{zu}$ entnehmen. Dann wird $z u$ jeder Konfigurations-,,Bezeichnung“ eine entsprechende Zeichnung angefertigt, und zwar in der oben besprochenen Weise [beginnend mit Kreis am Strich gefüllt ( $\bullet$ ) am C-Atom 1]. Aus diesen Zeichnungen läßt sich ablesen, welche Konfigurations-,,Bezeichnungen " für asymmetrische, welche für symmetrische und welche für identische Konfigurationen stehen. Bei der Übertragung der gefundenen Ergebnisse auf die aufgeschriebenen „Bezeichnungen" (Buchstabenkolonnen) wird durch Zusatz von a und b zu

9 Vgl. S. 163 der Mitt. XXX, l. c. ${ }^{3}$.

10 Für den Fall, daß die angegebenen Kriterien für die Zuordnung der Konfigurationen zu den Spalten „Körper“ und „Gegenkörper“ nicht ausreichen, haben wir den laufenden Nummern der „Bezeichnungen“ vermerkt, welche „Bezeichnungen“ für ein AntipodenPaar stehen (a für Bilder, b für Spiegelbilder). Dann wird ermittelt, welche Konfigurationen im Sinne einer gedachten Conversion ${ }^{9}$ zusammengehören. Die Conversions-Beziehungen sind wieder am leichtesten an Zeichnungen zu erkennen. Bei der Úbertragung dieser Beziehungen auf die „Bezeichnungen“ erfolgt dann die erste Auswahl der „Bezeichnungen“ für die provisorische Tabelle.

b) Umwandlung der provisorischen Konfigurations-Tabelle

Die provisorische Tabelle wird nach den in Mitt. $\mathrm{XXX}^{3}$ und $\mathrm{XXI}^{4}$ dieser Reihe gegebenen Richtlinien über die Zuordnung der Konfigurationen zu den Spalten „Körper“ und „Gegenkörper“ sowie über die Reihenfolge von e und a abgeändert. Dazu muß berücksichtigt werden: die Anzahl der metaStellungen a-gebundener Substituenten, die Anzahl der a-gebundenen Substituenten, die Anzahl der ortho-Stellungen e-gebundener Substituenten ${ }^{10}$. Danach läßt sich eine endgültige Tabelle aufstellen: Konfigurations-Tabellen ohne gesonderte Notierung optischer Antipoden. Durch Einführung der Antipoden-Schreibweise ${ }^{11}$ für asymmetrische Konfigurationen läßt sich die Konfigurations-Tabelle mit gesonderter Notierung optischer Antipoden erhalten.

\section{Beispiel zur Anwendung der Methode}

In Tab. 1, lfd. Nr. 1, sind die Bezeichnungen aller theoretisch möglichen Sessel-Konfigurationen des 1.2.4.5-Tetrabrom-1.4-dichlor-cyclohexans ohne, in Tab. 1, lfd. Nr. 2, mit gesonderter Notierung der optischen Antipoden zusammengestellt. Das Entstehen der genannten Tabellen ist mit Hilfe der nachstehenden Angaben aus Tafel 1 abzulesen. Es wurden nacheinander folgende Notizen gemacht:

a) Aufstellen der provisorischen Konfigurations-Tabelle

1. Aufschreiben aller Konfigurations„Bezeichnungen“, die sich für das als Beispiel gewählte Stellungsisomere, a usgehend von Buchstabenkolonne IV, der Tafel 2 finden lassen ${ }^{12}$ : Tafel 1 , A.

das berechnete Dipolmoment als weiteres Kriterium vorgeschlagen (vgl. Mitt. XXI, l. c. ${ }^{4}$ ).

11 Vgl. S. 166 der Mitt. XXX, 1. c. 3, und Mitt. XXI, 1. c. ${ }^{4}$.

12 Vgl. Erläuterungen und Beispiele zu Tafel 2. 


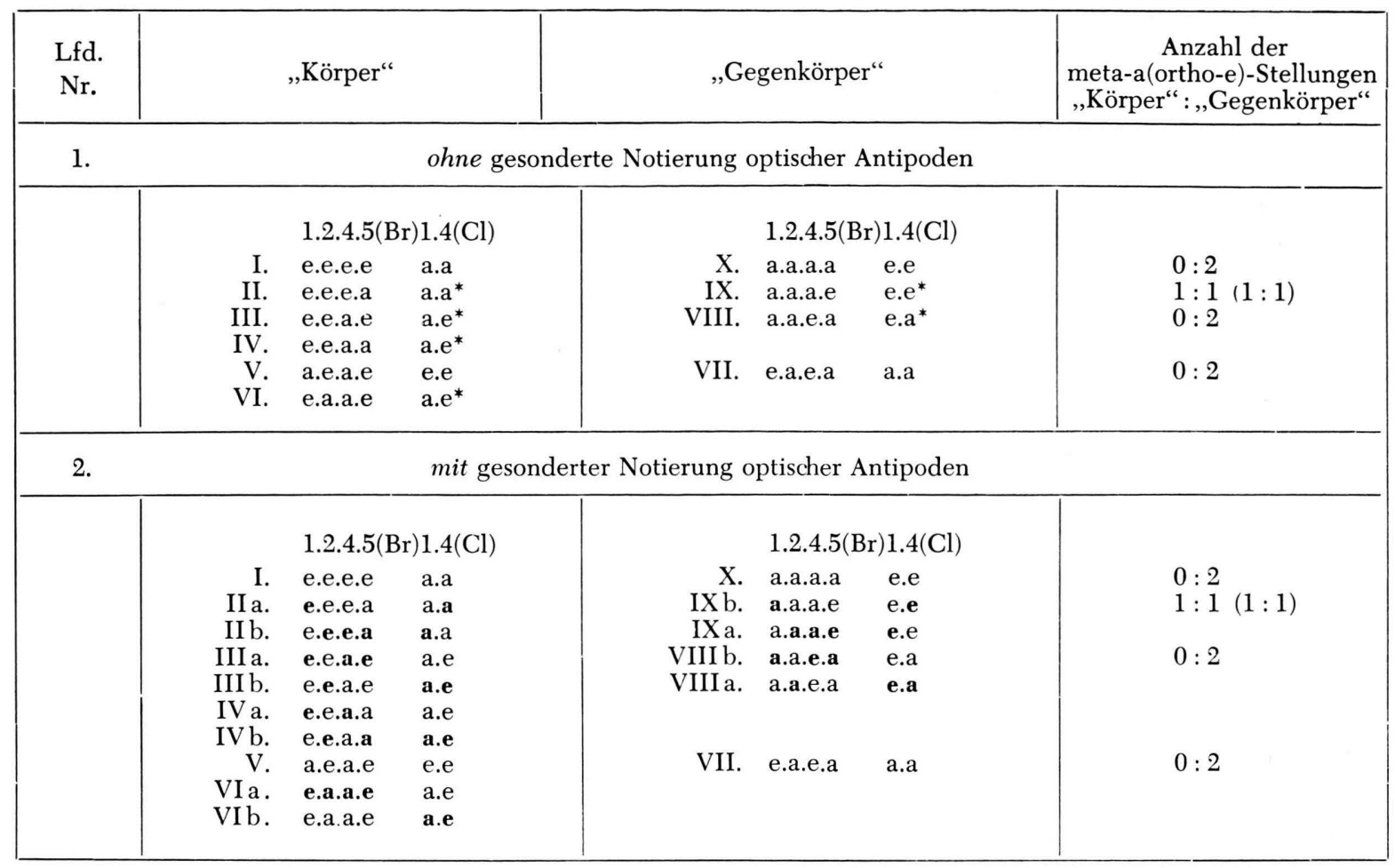

Tab. 1. Die theoretisch möglichen Sessel-Konfigurationen des 1.2.4.5-Tetrabrom-1.4-dichlor-cyclohexans.

Für das 1.2.4.5-Tetrabrom-1.4-dichlor-cyclohexan sind 16 Konfigurations-,,Bezeichnungen“ aufgeschrieben, und zwar stehen die Ziffern, die angeben, an welchen C-Atomen sich die Substituenten befinden, nur einmal über den Buchstaben e bzw. a. Durch Kombinieren der Ziffern mit den darunterstehenden Buchstaben lassen sich die vollständigen Konfigurations-,,Bezeichnungen“ ablesen. Die Konfigurations„Bezeichnungen“ sind mit arabischen Zahlen laufend durchnumeriert (laufende Nummer 1 bis 16).

2. Anfertigenderden Konfigurations„Bezeichnungen“ entsprechenden Konfigurations-Zeichnungen, unter Verwendung der in Abb. 1 erläuterten Darstellungsweise: Tafel 1, B.

Die Zeichnungen wurden den Konfigurations-,,Bezeichnungen “ entsprechend mit arabischen Zahlen numeriert. Die Zeichnungen wurden einheitlich angelegt, beginnend mit Kreis am Strich gefüllt $(\bullet)$ am C-Atom 1.

3. Heraussuchen bestimmter Zeichnungen, und zwar solcher, die symmetrische Konfigurationen darstellen. und solcher, die sich durch Drehen oder Klappen ${ }^{12 a}$ als Darstellung identischer bzw. zus a mmengehörender Bildund Spiegelbild-Konfigurationen erweisen: Tafel $1, B$.

Die Zeichnungen Nr. 1, 6, 11 und 16 (Tafel 1, B) stellen symmetrische Konfigurationen dar und wurden durch Umrandung ihrer Nummer hervorgehoben. Identische Konfigurationen sind im vorliegenden Beispiel nicht vorhanden. Die Zeichnung mit den Nummern 2 und 5, 3 und 9, 4 und 13, 7 und 10 , 8 und 14, 12 und 15 (Tafel 1, B) stellen jeweils zusammengehörende Bild- und Spiegelbild-Konfigurationen dar, was unter Verwendung des Zeichens $\%$ für Spiegelbild an den betreffenden Zeichnungen vermerkt wurde.

4. Übertragen der an den Zeichnungen (Tafel 1, B) gewonnenen Ergebnisse auf die "Bezeichnungen“ (Tafel 1, A): T a fel 1, C.

In Klammern ist vermerkt, welche „Bezeichnungen “ identische Konfigurationen angeben. Durch Zusatz von a für Bilder und $\mathrm{b}$ für Spiegelbilder $\mathrm{zu}$ den Nummern der „Bezeichnungen“ für asymmetrische 1.a Vgl. Text zur Abb. 1. 


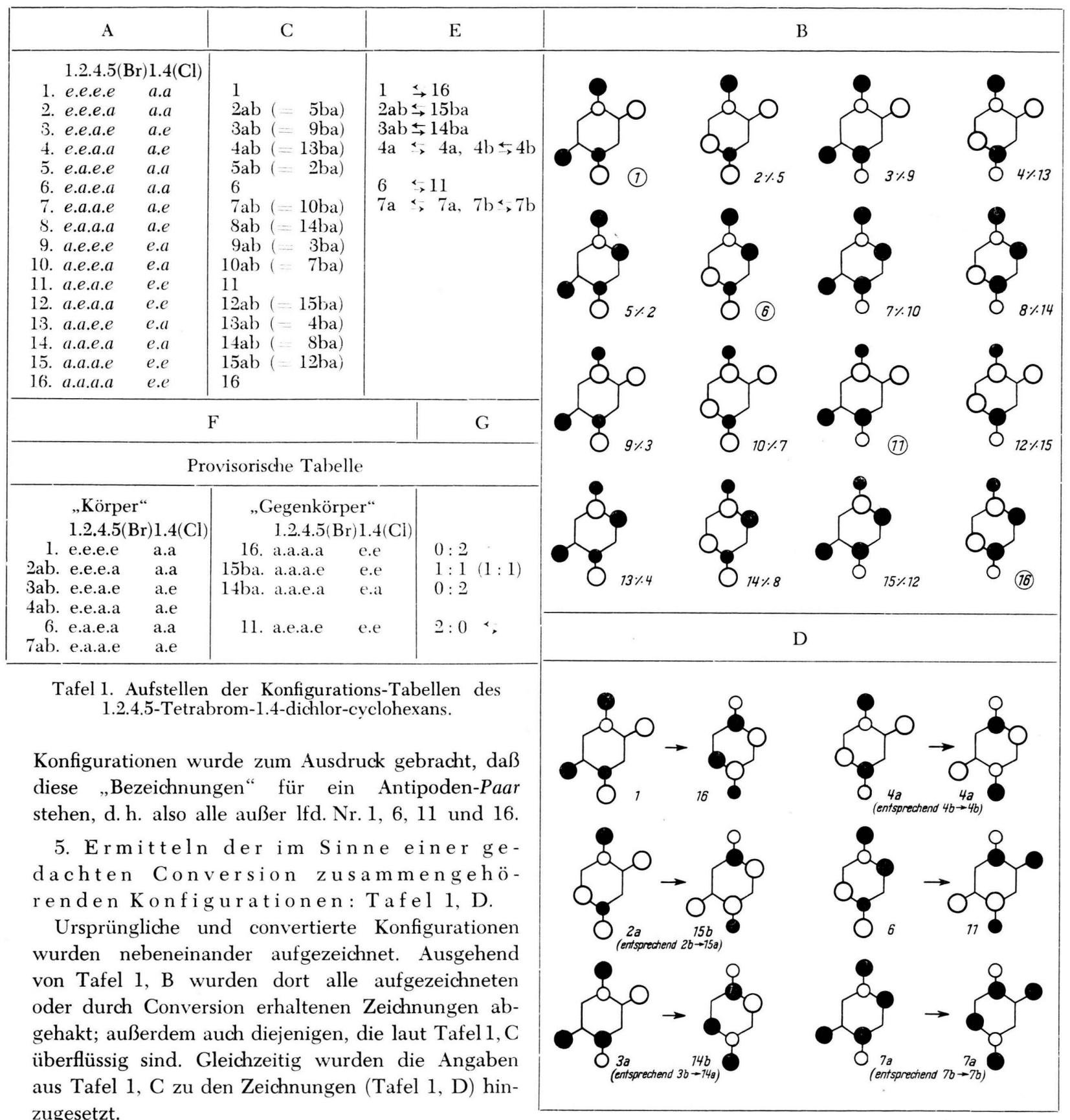

6. Übertragen der an $\mathrm{H}$ and der Zeichnungen (Tafel 1, D) gefundenen Conversions-Beziehungen auf die Konfigurations-, Bezeichnungen “ (Tafel 1, A): $\mathrm{T}$ a f e l 1, E.

Die unter den einzelnen Zeichnungen (Tafel 1, D) bereits notierten Beziehungen wurden zu den Konfigurations-,,Bezeichnungen“ (Tafel 1, A) hinzuge-

schrieben. Dadurch, daß die „Bezeichnungen“, die für identische Konfigurationen stehen, nicht mehr berücksichtigt wurden (Tafel $1, \mathrm{C}$ ), ist eine Auswahl der „Bezeichnungen“ für die provisorische Tabelle getroffen.

7. Aufschreiben der provisorischen Tabelle: Tafel 1, F. 
Die aus den bisherigen Notizen (besonders Tafel 1,E) bereits erkennbare Tabelle ist in zweispaltiger Form übersichtlich aufgeschrieben.

b) Umwandlung der provisorischen Konfigurations-Tabelle

8. Prüfen, ob die gewählten Bezeichnungen den in Mitt. XXX ${ }^{3}$ und $X X X I^{4}$ aufgestellten Richtlinien entsprechen, und zwar in bezug auf Zuordnung zu den Spalten „Körper“ und „Gegenkörper" und in bezug auf die Reihenfolgevone und a, und Angabe dernotwendigen Änderungen: Tafel 1, G.

Die Anzahl der meta-Stellungen a-gebundener Substituenten, die Anzahl der a-gebundenen Substituenten und die Anzahl der ortho-Stellungen egebundener Substituenten lassen sich aus den Zeichnungen (Tafel $1, D)$ ohne weiteres ablesen und sind zur provisorischen Tabelle (Tafel 1, F) dazugeschrieben: Tafel 1, G. Wegen der Anzahl der meta-a-Stellungen sind die Konfigurationen 1e2a4e $5 \mathrm{a}(\mathrm{Br}) 1 \mathrm{a} 4 \mathrm{a}(\mathrm{Cl})$ und 1a2e4a5e(Br)1e4e(Cl) umzustellen, was ebenfalls in der provisorischen Tabelle (Tafel $1, F$ ) vermerkt ist (Tafel 1, G). In bezug auf die Reihenfolge von e und a sind im vorliegenden Beispiel keine Änderungen nötig.

9. Aufschreiben einer endgültigen Tabelle.

Wenn man alle Änderungen vorgenommen, die Konfigurations-Bezeichnungen mit römischen Zahlen neu durchnumeriert und alle durch den Zusatz a und $\mathrm{b}$ als für ein Antipoden-Paar stehend gekennzeichneten Bezeichnungen mit einem Sternchen versehen hat, resultiert die Konfigurations-Tabelle ohne gesonderte Notierung optischer Antipoden: Tab. 1, lfd. Nr. 1. Um zur Konfigurations-Tabelle mit gesonderter Notierung optischer Antipoden (Tab. 1, lfd. Nr. 2) zu gelangen, wird genau so mit römischen Zahlen durchnumeriert, d. h. ein Antipoden-Paar bekommt nur eine gemeinsame Nummer. Dann wird für asymmetrische Konfigurationen die AntipodenPaar-Schreibweise gemäß Mitt. XXX ${ }^{11}$ in die Antipoden-Schreibweise umgewandelt, und die Bezeichnungen werden nach Maßgabe der unter Tafel 1, E angegebenen Beziehungen zwischen Bild- und Spiegelbild-Konfigurationen aufgeschrieben. Bild bzw. Spiegelbild werden durch Zusatz von a bzw. b zu ihrer laufenden Nummer noch zusätzlich gekennzeichnet.
Man kann natürlich die Tabelle mit gesonderter Notierung optischer Antipoden auch direkt aus der Tabelle ohne gesonderte Notierung optischer Antipoden entwickeln, muß dann aber die ConversionsBeziehungen von solchen optischen Antipoden nachprüfen, deren Bezeichnung in Antipoden-PaarSchreibweise nur in der Spalte „Körper“ steht. Es ist einfacher, auf die Notizen unter Tafel 1, E zurückzugreifen.

Bei der Durchführung anderer Beispiele können einige Besonderheiten zutage treten. Zum Beispiel können alle nach der gegebenen Anleitung aufgezeichneten Konfigurationen asymmetrisch sein, worunter z. B. beim 1.2-Dibrom-4.5-dichlor-cyclohexan zwar identische asymmetrische Konfigurationen, aber keine Antipodenpaare sind. In einem solchen Falle müssen, wie immer, die identischen Konfigurationen sehr sorgfältig herausgesucht werden, und es darf nicht vergessen werden anzumerken, daß alle aus den Zeichnungen ablesbaren Bezeichnungen dann für. Antipoden-Paare stehen.

Wenn nicht eine Konfigurations-Tabelle, sondern nur die Anzahl der theoretisch möglichen Konfigurationen und die auftretenden Arten der ConversionsBeziehungen ${ }^{13}$ ermittelt werden sollen, ist eine KurzTabelle recht brauchbar, in der keine KonfigurationsBezeichnungen, sondern nur die römischen Nummern mit den Zusätzen a und b für Bild und Spiegelbild stehen. Die zum besprochenen Beispiel gehörende Kurz-Tabelle sieht folgendermaßen aus:

I $\leftrightarrows \mathrm{X}$
II a $\leftrightarrows$ IX b
II $\mathrm{b} \leftrightarrows$ IX a
III a $\leftrightarrows$ VIII b
III $\leftrightarrows$ VIII a
IV a
IVb
V $\leftrightarrows$ VII
VI a
VIb

Die bei der Vorführung des Beispiels der Deutlichkeit halber sehr auseinandergezogene Darstellung läßt sich vereinfachen: Wenn erst eine gewisse Übung vorhanden ist, werden die hier einzeln geschilderten Schritte z. T. gleichzeitig ausgeführt werden können, vor allem läßt sich die Schreibarbeit wesentlich reduzieren, wenn bei der ersten Aufschreibung der Konfigurations-, ,Bezeichnungen“(Buchstabenkolonne) und neben den ersten Zeichnungen genügend Platz gelassen wird, um nötige Änderungen durch Streichen und Daneben- oder Darüber-

$13 \mathrm{Vgl}$. die Übersicht auf Seite 391 der Mitt. XXVIII, 1. c. ${ }^{14}$. 


\begin{tabular}{|c|c|c|c|c|c|c|c|c|c|c|c|c|}
\hline \multirow{3}{*}{$\begin{array}{l}\text { Lfd. } \\
\text { Nr. }\end{array}$} & & & & \multicolumn{8}{|c|}{ Anzahl der vereinfacht ebenen und Sessel-Konfigurationen } & \multirow{3}{*}{$\begin{array}{l}\text { Konf. } \\
\text { - Tab. }\end{array}$} \\
\hline & \multicolumn{3}{|c|}{ Stellung der Substituenten } & \multirow{2}{*}{\multicolumn{2}{|c|}{$\begin{array}{c}\text { „Körper“ } \\
\text { gleichzeitig } \\
\text { vereinfacht eben } \\
\text { B. }(+ \text { Sp. })\end{array}$}} & \multirow{2}{*}{+} & \multirow{2}{*}{\multicolumn{2}{|c|}{$\begin{array}{l}\text { „Gegenkörper“ } \\
\text { B. (+ Sp.) }\end{array}$}} & \multicolumn{3}{|c|}{$\begin{array}{c}\text { „Körper“ + } \\
\text { „Gegenkörper“ }\end{array}$} & \\
\hline & $\mathrm{Br}$ & $\mathrm{Cl}$ & $\mathrm{F}$ & & & & & & B. & $(+$ Sp. $)$ & insges. & \\
\hline 1 & 1 & 4 & & 1 & & + & 1 & & 2 & & 2 & $\mathrm{c}$ \\
\hline 2 & 1 & 1.2 & & 2 & $(+2)$ & + & 2 & $(+2)$ & $\overrightarrow{4}$ & $(+4)$ & 8 & c \\
\hline 3 & 1.2 & 1 & & 2 & $(+2)$ & & 2 & $(+2)$ & 4 & $(+4)$ & 8 & c \\
\hline 4 & 1 & 1.4 .4 & & 1 & & + & 1 & & 2 & & 2 & c \\
\hline 5 & 1 & 2.4 .5 & & 8 & $(+8)$ & + & 8 & $(+8)$ & 16 & $(+16)$ & 32 & c \\
\hline 6 & 1.2 & 3.4 & & 8 & $(+8)$ & + & 8 & $(+8)$ & 16 & $(+16)$ & 32 & c \\
\hline 7 & 1.2 & 3,6 & & 6 & $(+4)$ & + & 4 & $(+6)$ & 10 & $(+10)$ & 20 & c \\
\hline 8 & 1.2 & 4.5 & & 6 & $(+4)$ & + & 4 & $(+6)$ & 10 & $(+10)$ & 20 & $\mathrm{e}$ \\
\hline 9 & 1.3 & 2.4 & & 8 & $(+8)$ & + & 8 & $(+8)$ & 16 & $(+16)$ & 32 & c \\
\hline 10 & 1.3 & 4.6 & & 6 & $(+4)$ & + & 4 & $(+2)$ & 10 & $(+6)$ & 16 & c \\
\hline 11 & 1.4 & 2.3 & & 6 & $(+4)$ & + & 4 & $(+6)$ & 10 & $(+10)$ & 20 & c \\
\hline 12 & 1.4 & 2.5 & & 6 & $(+4)$ & + & 4 & $(+2)$ & 10 & $(+6)$ & 16 & e \\
\hline 13 & 1.2 .4 & 5 & & 8 & $(+8)$ & + & 8 & $(+8)$ & 16 & $(+16)$ & 32 & $\mathrm{c}$ \\
\hline 14 & 1.1 .4 & 4 & & 1 & & + & 1 & & 2 & & 2 & c \\
\hline 15 & 1 & 2.3.4.5.6 & & 20 & $(+12)$ & + & 20 & $(+12)$ & 40 & $(+24)$ & 64 & c \\
\hline 16 & 1.2 & 3.4.5.6 & & 20 & $(+16)$ & + & 16 & $(+20)$ & 36 & $(+36)$ & 72 & e \\
\hline 17 & 1.3 & 2.4.5.6 & & 20 & $(+12)$ & + & 20 & $(+12)$ & 40 & $(+24)$ & 64 & c \\
\hline 18 & 1.4 & 1.2 .4 .5 & & 6 & $(+4)$ & + & 4 & $(+2)$ & 10 & $(+6)$ & 16 & c \\
\hline 19 & 1.4 & 2.2 .5 .5 & & 2 & $(+1)$ & + & 1 & & 3 & $(+1)$ & 4 & c \\
\hline 20 & 1.4 & 2.3.5.6 & & 14 & $(+6)$ & + & 10 & $(+6)$ & 24 & $(+12)$ & 36 & $\mathrm{e}$ \\
\hline 21 & 1.1 .4 .4 & 2.5 & & 2 & $(+1)$ & + & 1 & & 3 & $(+1)$ & 4 & c \\
\hline 22 & 1.2 .3 .4 & 5.6 & & 20 & $(+16)$ & + & 16 & $(+20)$ & 36 & $(+36)$ & 72 & c \\
\hline 23 & 1.2 .3 .5 & 4.6 & & 20 & $(+12)$ & + & 20 & $(+12)$ & 40 & $(+24)$ & 64 & c \\
\hline 24 & 1.2 .4 .5 & 1.4 & & 6 & $(+4)$ & + & 4 & $(+2)$ & 10 & $(+6)$ & 16 & f \\
\hline 25 & 1.2 .4 .5 & 3.6 & & 14 & $(+6)$ & + & 10 & $(+6)$ & 24 & $(+12)$ & 36 & c \\
\hline 26 & 1 & 1.2 .3 .4 .5 .6 & & 20 & $(+12)$ & + & 20 & $(+12)$ & 40 & $(+24)$ & 64 & c \\
\hline 27 & 1.2 & 1.3 .4 .5 .6 & & 32 & $(+32)$ & + & 32 & $(+32)$ & 64 & $(+64)$ & 128 & $\mathrm{c}, \mathrm{g}$ \\
\hline 28 & 1.1 .2 & 3.4 .5 .6 & & 16 & $(+16)$ & + & 16 & $(+16)$ & 32 & $(+-32)$ & 64 & $c^{\circ}$ \\
\hline 29 & 1.2 .3 & 1.4 .5 .6 & & 32 & $(+32)$ & + & 32 & $(+32)$ & 64 & $(+64)$ & 128 & $\mathrm{c}, \mathrm{g}$ \\
\hline 30 & 1.2 .3 .4 & 1.5 .6 & & 32 & $(+32)$ & + & 32 & $(+32)$ & 64 & $(+64)$ & 128 & $c, g$ \\
\hline 31 & 1.2 .3 .4 & 5.5 .6 & & 16 & $(+16)$ & + & 16 & $(+16)$ & 32 & $(+32)$ & 64 & c \\
\hline 32 & 1 & 1.2 .3 .4 .4 .5 .6 & & 10 & $(+6)$ & + & 10 & $(+6)$ & 20 & $(+12)$ & 32 & $\mathrm{c}$ \\
\hline 33 & & 1.2 .3 .4 .5 .6 & 1 & 20 & $(+12)$ & + & 20 & $(+12)$ & 40 & $(+24)$ & 64 & c \\
\hline 34 & & 1.1 .2 .3 .4 .5 .6 & 4 & 10 & $(+6)$ & + & 10 & $(+6)$ & 20 & $(+12)$ & 32 & c \\
\hline 35 & 1.2 & 3.4 .5 .6 & 1 & 32 & $(+32)$ & + & 32 & $(+32)$ & 64 & $(+64)$ & 128 & $\mathrm{c}, \mathrm{g}$ \\
\hline 36 & 1.2 & 3.4 .5 .6 & 3 & 32 & $(+32)$ & + & 32 & $(+32)$ & 64 & $(+64)$ & 128 & $\mathrm{c}, \mathrm{g}$ \\
\hline 37 & 1.2 & 3.4 .5 .6 & 4 & 32 & $(+32)$ & + & 32 & $(+32)$ & 64 & $(+64)$ & 128 & c, g \\
\hline
\end{tabular}

Tab. 2. Zahlenmäßige Verteilung der Sessel-Konfigurationen einiger Polyheterohalocyclohexane auf „Körper“ und „Gegenkörper“ unter getrennter Berücksichtigung der Bilder (B.) a und Spiegelbilder (Sp.) ${ }^{\text {b. }}$

a Unter B. zählen wir die Bilder der asymmetrischen Konfigurationen und dazu die symmetrischen Konfigurationen (bei denen wir ja keine Bilder und Spiegelbilder im Sinne des Chemikers unterscheiden können).

b Unter Sp. sind die Spiegelbilder gesondert aufgeführt, womit also gleichzeitig die Anzahl der Antipodenpaare angegeben ist.

c Unveröffentlicht.

d Die Zahl der vereinfacht ebenen Konfigurationen und der Sessel-Konfigurations-Paare ist identisch. Diese

schreiben vornehmen und die Conversions-Partner danebenzeichnen zu können.

Aus Konfigurations-Tabellen mit getrennter Notierung optischer Antipoden und auch aus Kurz-Tabellen läßt sich die zahlenmäßige Verteilung der zu
Zahl ist in der Spalte „Körper“ zu finden, da wir in den Fällen, in denen eine Sessel-Konfiguration bei Conversion in sich selbst übergeht, diese Konfiguration unter „Körper“ gezählt haben.

e Tab. 2 der Mitt. XXIII, l. c. ${ }^{1}$.

f Tab. 1 dieser Arbeit.

g Mehr als 128 Sessel-Konfigurationen sind für ein Stellungsisomeres von Cyclohexan-Substitutionsprodukten niemals möglich. Vergleiche den letzten Absatz der Erläuterungen zu Tafel 2.

einem Stellungsisomeren gehörenden theoretisch möglichen Sessel-Konfigurationen auf die Spalten „Körper" und "Gegenkörper" unter Berücksichtigung der Bilder und Spiegelbilder ablesen. Für eine größere Anzahl von Stellungsisomeren sind solche zahlen- 
mäßigen Verteilungen der zugehörigen Sessel-Konfigurationen in Tab. 2 dieser Arbeit und in Tab. 3 der Mitt. XXVIII ${ }^{14}$ wiedergegeben. In diesen Tabellen sind unter B. die Bilder der asymmetrischen Konfigurationen (in Konfigurations-Tabellen römische Nummern mit Zusatz a) und dazu die symmetrischen Konfigurationen (in Konfigurations-Tabellen römische Nummern ohne Zusatz von a oder b) gezählt, unter Sp. die Spiegelbilder der asymmetrischen Konfigurationen (in Konfigurations-Tabellen römische Nummern mit Zusatz b).

\section{Erläuterungen zu Tafel 2}

Unter I bis VI sind alle durch Kombinieren der Buchstaben $\mathrm{e}$ und a aufschreibbaren "Bezeichnungen“ für Sessel-Konfigurationen von Cyclohexan-Substitutionsprodukten mit einem bis sechs untereinander gleichen Substituenten, die sich an verschiedenen C-Atomen befinden, aufgeschrieben ". Bei diesen Konfigurations-,,Bezeichnungen" handelt es sich natürlich nicht um endgültige. Um den Unterschied zwischen solchen provisorischen Aufschreibungen und endgültigen Konfigurations-Bezeichnungen ${ }^{b}$ zum Ausdruck zu bringen, werden in vorliegender Arbeit alle provisorischen „Bezeichnungen“ kursiv gedruckt $^{\mathrm{C}}$. Die oben angegebenen sechs Buchstabenkolonnen lassen sich als solche oder nach entsprechender Ergänzung auch für die Aufschreibung von Sessel-Konfigurationen aller anderen Cyclohexan-Substitutionsprodukte verwenden. Die Entscheidung, welche der sechs Buchstabenkolonnen man für ein gegebenes Stellungsisomeres nehmen kann bzw. von welcher man ausgehen muß, hängt nicht von der Zahl der Substituenten ab, sondern von der Zahl der C-Atome, die mit einem Substituenten und einem H-Atom (CHX) bzw. mit zwei verschiedenen Substituenten (CXY) besetzt sind. $\mathrm{CX}_{\text {o }}$ Gruppen spielen zunächst keine Rolle, da für zwei gleiche an einem C-Atom befindliche Substituenten nur ea möglich ist. Bezüglich der gegebenenfalls erforderlichen Ergänzung ist zu sagen, daß die gewählte Buchstabenkolonne entweder durch Zusatz von einer oder mehreren ea-Reihen ergänzt wird (für $\mathrm{CX}_{\text {, }}$-Gruppen) oder daß $\approx u$ ea hin ergänzt wird (für $\mathrm{CXY}$ wird $\mathrm{X}$ aus der Buchstabenkolonne festgelegt und für $\mathrm{Y}$ zu ea hin ergänzt). Vergleiche die Beispiele zu Tafel 2.

Die Anzahl der für ein Stellungisomeres aufschreibbaren Sessel-Konfigurations-,,Bezeichnungen“" beträgt $2^{n}$, wobei $n$ die Zahl der mit einem Substituenten und einem H-Atom (CHX) bzw. mit zwei verschiedenen Substituenten besetzten C-Atome (CXY) ist. Da $n$ nicht größer als 6, d. h. nicht größer als die Anzahl der überhaupt vorhandenen C-Atome sein kann, beträgt die größtmögliche Anzahl von aufschreibbaren Konfigurations..Bezeichnungen“" dieser Art $2^{6}=64$.

Da bei der in dieser Tafel benutzten Art der Aufschreibung optische Antipoden nicht berücksichtigt sind und jede Bezeichnung gegebenenfalls für ein AntipodenPaar steht, kann für den Fall, daß nach Umwandlung in die Antipoden-Schreibweise keine identischen Konfigurationen aufgeschrieben sind, die Zahl der theoretisch möglichen Konfigurationen doppelt so groß sein, d. h. im Höchstfalle 128 betragen. In einem solchen Faile liegt nur Conversions-Möglichkeit Ia $\leftrightarrows$ IIb. Ib $\leftrightarrows$ IIa vor d. Beispiele vergleiche Tab. 2, Ifd. Nr. $27,29,30,35$. 36. 37.

\begin{tabular}{|c|c|c|c|}
\hline 1 & 15. a. & 31. a.a.a.a.e & 31. e.a.a.a.a.e \\
\hline 1. $e$ & 16. a.a.a.a & 32. a.a.a. & 32. e.a.a.a.a.a \\
\hline 2. $a$ & $V$ & 1 & 33. a.e.e.e.e \\
\hline II & 1. e.e & 1. е.е.е.е.е.е & 35. a.e.e.e.e.a \\
\hline 1. e.e & 2. e.e.e.e.a & 2. e.e.e.e.e.a & 36. a.c.e.e.a.a \\
\hline 2. e.a & 3. e.e.e.a.e & 3. e.e.e.e.a.e & 37. a.e.e.a.e.e \\
\hline 3. a.e & 4. e.e.e.a.a & 4. e.e.e.e.a.a & 38. a.e.e.a.e.a \\
\hline 4. a.a & 5. e.e.a.e.e & 5. e.e.e.a.e.e & 39. a.e.e.a.a.e \\
\hline III & 6. e.e.a.e.a & 6. e.e.e.a.e.a & 40. a.e. \\
\hline 1. e.e.e & 7. e.e.a.a.e & 7. e.e.e.a.a.e & 41. a.e.a.e.e.e \\
\hline 2. e.e.a & 8. e.e.a.a.a & 8. е.е.е.а.а.а & 42. а.е.а.е.е.а \\
\hline 3. e.a.e & 9. e.a.e.e.e & 9. е.е.а & 43. a.e.a.e.a.e \\
\hline 4. e.a.a & 10. e.a. & 10. e.e.a. & 44. a.e \\
\hline 5. a.e.e & 1. $e . a$ & 11. e.e.a & 45. a.e \\
\hline 6. a.e.a & 2. $e . a$ & 12. e.e.a. & 46. a.e.d \\
\hline 7. a.a.e & 13. e.a. & 13. e.e.a.a.e.e & 47. a.e.a.a.a.e \\
\hline 8. a.a.a & $e . a$ & 14. e.e.c & 48. а.е.а.а.а.а \\
\hline & . $e .0$ & 15. $e$. & 49. \\
\hline 15 & 16. e.a & 16. & $2 . a$ \\
\hline 1. e.e.e.e & 17. a.e & 17. e.a & 51. \\
\hline 2. e.e.e.a & 1S. a.e & 18. e.a. & 52. a. \\
\hline 3. e.e.a.e & 19. а.е.е.а.e & 19. е.а.е.е.а.е & 53. a.c \\
\hline 4. e.e.a.a & 20. a.e.e.a.a & 20. e.a.e.e.a.a & 54. a.c \\
\hline 5. e.a.e.e & 21. a.e.a.e.e & 21. e.a.e.a.e.e & 55. а.а.e.a.a.e \\
\hline 6. e.a.e.a & 22. а.е.а.е.a & 22. е.а.е.а.е.a & 56. а.а.e.a.a.a \\
\hline 7. e.a.a.e & 23. а.e.a.a.e & 23. e.a.e.a.a.e & 57. a.a.a.e.e.e \\
\hline S. e.a.c & 24. а.е.а.а.а & 24. e.a.e.a.a.a & 58. a.a.a \\
\hline 9. a.e.e & 25. a.a.e.e.e & 25. e.a.a.e.e.e & 59. a.a.a.e.a.e \\
\hline 10. a.e.e. & 26. a.a.e.e.a & 26. e.a.a.e.e.a & 60. a.a.a.e.a.a \\
\hline 11. a.e.a.e & 27. a.a.e.a.e & 27. e.a.a.e.a.e & 61. a.a.a.a.e.e \\
\hline 12. a.t & 28. а.а.e.a.a & 25. е.а.а.е.а.а & 62. a.a.a.a.e.a \\
\hline a. & a.a.a.e.e & 29. e.a.a.a.e.e & 63. a.a.a.a.a.e \\
\hline 4. a.a & 30. a.a.a.e.a & 30. e.a.a.a.e.a & 64. a.a.a.a.a.a \\
\hline
\end{tabular}

Tafel 2. Über das Notieren aller durch Kombinieren von e und a aufschreibbaren „Bezeichnungen“ für SesselKonfigurationen von Cyclohexan-Substitutionsprodukten.

Beispiele zur Tafel 2

1.1.2.4.4.5 1.1.2.4.4.5

$\begin{array}{ll}\text { 1. ea.e.ea.e } & \text { 3. ea.a.ea.e } \\ \text { 2. ea.e.ea.a } & \text { 4. ea.a.ea.a }\end{array}$

Die vorstehend angegebene Aufschreibung aller Sessel-Konfigurations-,,Bezeichnungen“ für das Stellungsisomere 1.1.2.4.4.5 ist $\mathrm{zu}$ erhalten durch Kombinieren der Buchstabenkolonne II der Tafel mit zwei Reihen ea für die CX.,-Gruppen der C-Atome 1 und 4.

\begin{tabular}{|c|c|c|c|c|}
\hline \multicolumn{2}{|c|}{$1.2 .4 .5(\mathrm{X}) 1.4(\mathrm{Y})$} & \multicolumn{3}{|c|}{$1.2 .4 .5(\mathrm{X}) 1.4(\mathrm{Y})$} \\
\hline 1. e.e.e.e & a.a & 9. & a.e.e.e & $e . a$ \\
\hline . e.e.e.a & a.a & 10. & a.e.e.a & e.a \\
\hline e.e.a.e & a.e & 11. & a.e.a.e & e.e \\
\hline e.e.a.a & a.e & 12. & a.e.a.a & e.e \\
\hline 5. e.a.e.e & a.a & 13. & a.a.e.e & e.a \\
\hline 6. e.a.e.a & a.a & 11. & a.a.e.a & e.a \\
\hline 7. e.a.a.e & a.e & 15. & a.a.a.e & e.e \\
\hline 8. e.a.a.a & a.e & 16. & a.a.a.a & e.e \\
\hline
\end{tabular}

Um zu der vorstehend angegebenen Aufschreibung aller Sessel-Konfigurations-,,Bezeichnungen “ für das Stellungsisomere 1.2.4.5 (X)1.4 (Y) zu gelangen. muß man von Buchstabenkolonne IV der Tafel ausgehen. Damit sind

14 Angew. Chem. 65, 390, 627 [195:3]. 
die in 1.4-Stellung befindlichen Substituenten $\mathrm{X}$ festgelegt; um die Lage der Substituenten Y an den C-Atomen 1 und 4 angeben zu können, ist jeweils nach ea hin ergänzt worden.

\begin{tabular}{ll} 
1.1.2.4 $(\mathrm{X}) 2.5(\mathrm{Y})$ & \multicolumn{2}{c}{$1.1 .2 .4(\mathrm{X}) 2.5(\mathrm{Y})$} \\
1. ea.e.e a.e & 5. ea.a.e e.e \\
2. ea.e.e a.a & 6. ea.a.e e.a \\
3. ea.e.a a.e & 7. ea.a.a e.e \\
4. ea.e.a a.a & 8. ea.a.a e.a
\end{tabular}

Die vorstehende Aufschreibung aller Sessel-Konfigurations-,,Bezeichnungen“ für das Stellungsisomere 1.1.2.4(X)2.5(Y) wurde erhalten, indem zur Buchstabenkolonne
III der Tafel eine Reihe ea für die $C_{9}$-Gruppe am C-Atom 1 zugesetzt und für den Substituenten $Y$ am C-Atom 2 nach ea hin ergänzt wurde.

a Sieht man sich in den oben aufgeführten Buchstabenkolonnen die Reihen der untereinander stehenden Buchstaben an, so ist zu bemerken, daß in der letzten Reihe abwechselnd e und a untereinander stehen, in der vorletzten Reihe zwei e und zwei a, in der vorvorletzten Reihe doppelt so viel usw.

b (in Antipoden-Paar- oder in Antipoden-Schreibweise). Vgl. Mitt. XXX und XXI, l. c. ${ }^{3,4}$.

c Vgl. auch Tafel 1.

d Vgl. die Übersicht auf S. 391 der Mitt. XXVIII, l.c. ${ }^{14}$.

\title{
Polysulfonsäure-Derivate mit Antivirus-Wirkung
}

\author{
Von R. Neher und F. Kradolfer \\ Aus den wissenschaftlichen Laboratorien der Ciba Aktiengesellschaft Basel \\ (Z. Naturforschg. 10 b, 191-198 [1955]; eingegangen am 28. Dezember 1954)
}

1. Verschiedene aromatische Polysulfonsäure-Derivate (P.S.) zeigen sowohl an der Chorioallantois als auch am Brutei eine Antivirus-Wirkung, die sich gegenüber Stämmen von Influenzavirus und New Castle Disease-Virus nachweisen läßt.

2. Versuche zur Analyse des Antiviruseffektes ergaben, daß die untersuchten Polysulfonsäuren das Virus entweder vor dessen Eintritt in die Gewebszellen oder in frühen Stadien der Invasionsphase erreichen müssen, um eine volle Wirkung auszuüben. Auch die Möglichkeit einer Hemmung der Liberationsphase, wie sie bei anderen Antivirusstoffen bekannt ist, ist für die P.S. nicht ausgeschlossen.

3. Die meisten der untersuchten Polysulfonsäuren hemmen die Virushämagglutination in vitro. Mit RDE vorbehandelte Erythrozyten, die arm an Virusrezeptoren sind, werden durch dieselben Konzentrationen von P.S. agglutiniert wie normale Erythrozyten. Der Aspekt der Agglutination läßt sich unterscheiden von der Form der Virushämagglutination. Es ist deshalb anzunehmen, daß die P.S. nicht ausschließlich am Virusrezeptor der Erythrozyten angreifen.

Ein Antagonismus zwischen P.S. und bekannten Virusrezeptor-Substanzen (Ovomucin, Mucoprotein) konnte nicht nachgewiesen werden. Auf Grund dieser Versuche wird vermutet, daß die P.S. die Bindung des Virus an das spezifische Substrat beeinflussen und daß dafür im wesentlichen physikalisch-chemische Reaktionen an Grenzflächen maßgebend sind.

4. Bei wiederholter Anwendung können die P.S. zu intrazellulären Veränderungen an Mesenchymzellen führen, doch ist nicht anzugeben, inwiefern diesem Effekt eine Bedeutung für die Antivirus-Wirkung zukommt.

5. Verschiedene P.S. mit Antiviruseffekt hemmen die Blutgerinnung und Immunhämolyse und lösen das Zellkern-Quellungsphänomen aus, während P.S. mit geringer oder fehlender Antivirus-Wirkung diese Eigenschaften nicht oder weniger deutlich aufweisen. Solche Beziehungen zwischen Antivirusaktivität und diesen „Nebenwirkungen“ gelten offenbar nicht für alle Derivate vom Charakter der Polysulfonsäuren.

$\mathrm{V}$

or einigen Jahren haben wir eine neue, gegenüber Influenza-Virus wirksame Stoffgruppe aufgefunden, die inzwischen chemisch und biologisch weiterbearbeitet worden ist $\left(\mathrm{Kradolf} \mathrm{er}^{1}\right)$. Auch von anderen Autoren ist über den Effekt von sulfonsäure-haltigen Verbindungen gegenüber Virusinfektionen berichtet worden $\left(\mathrm{Hurst}{ }^{2}\right.$, T a kem ot ${ }^{3}$,

1 F. Kradolfer, Schweiz. Z. Path. u. Bakt. 17, 520 [1954].

2 E. W. Hurst, Brit. J. Pharmacol. Chemotherapy 7,455 [1952].
Ackermann ${ }^{4}$ ). Die vorliegende Mitteilung gibt einen Überblick über die von uns durchgeführten Untersuchungen.

\section{Chemische Eigenschaften}

Es handelt sich um aromatische Sulfonsäuren, die durch Harnstoff-, Triazin- oder ähnliche polyfunktio-

3 K. K. T a k e m o to, J. Immunology 72, 139 [1954].

4 W. W. A ckermann u. H. F. M a s s a b, J. exp. Medicine 99, 121, 105 [1954]. 